cells in the midbrain. These neurones thus seem to act like feature detectors, and by comparing the spectral composition of echoes with the emitted signals, they may serve to detect the size and location of the reflecting object.

The study of the bat's auditory system is providing us with unexpected insights into how the brain is designed to utilise biosonar signals. The next task for researchers is to elucidate the intricate neural network that enables

\section{Positrons at surfaces}

\section{from John Pendry}

RADIOACTIVE sources provide a supply of positrons in the laboratory, and one of the applications of these particles is to studies of the solid state. In a recent Letter (Mills, Platzman \& Brown, Phys. Rev. Lett. 41, 1076; 1978) an important new advance in technique has been demonstrated: positrons can be controlled precisely enough to probe the surface of a solid on an atomic scale.

Conventional experiments use positrons straight from the source with energies of the order of an $\mathrm{MeV}$, and fire them into the solid under study. Once inside, a positron interacts strongly with the electrons through Coulomb forces, Plasmons, collective oscillations of the electrons, rapidly dissipate the energy and in a very short time the positron has lost almost all its energy and momentum. Capture of the positron by its antiparticle, the electron, will eventually take place, producing a pair of $\gamma$ rays with the disappearance of the positron and an electron, though the time for annihilation is long compared with the time to come to rest in the solid. By conservation of momentum the $\gamma$ rays must have almost oppositely directed momenta, slight deviations from $180^{\circ}$ being due to the relatively small but finite moments of the electrons with which the positrons annihilate. Measurement of the deviation, in experiments which look for coincidences of two $\gamma$ rays, gives the momentum distribution of electrons in the solid, information which can be used to understand bonding of solids, and to measure the Fermi surfaces of metals.

An additional complication arises for insulators, where the positron's Coulomb potential is largely unscreened, and is strong enough to bind an electron into a hydrogenic state known as positronium. The positron will preferentially annihilate with its

John Pendry is at the Daresbury Laboratory of the Science Research Council. certain features of acoustic stimuli to be extracted, and the processes by which such connections evolve during development. Moreover, it will be of great interest to know how the middle ear of the animal is constructed so as to vibrate optimally at ultrasonic frequencies. Once this task is achieved, we shall be a step nearer to understanding the mechanisms of sensory processing and integration, for which the brain is most exquisitely designed.

'own' electron, and signals characteristic not of the solid, but of the positronium bound state, are seen.

Surface sensitivity in positron experiments came a step nearer when it was shown that the thermalised positrons can be re-emitted from a solid before they have time to annihi1ate. Unlike electrons, positrons tend to be repelled by many solids and if in diffusing around the solid they encounter a surface they will be expelled from the solid. The positrons do not acquire more than about $1 \mathrm{eV}$ energy so are reasonably monochromatic and can be accelerated by electric fields to form a collimated beam with easily controlled energy (Canter, Mills \& Berko Phys. Rev. Lett. 33, 7; 1974).

The point is that positrons with only $100 \mathrm{eV}$ kinetic energy have limited powers of penetration, typically a few tens of $\AA$, and therefore can be used to probe surfaces on an atomic scale.

Mills, Platzman and Brown have performed the first experiments of this nature. They worked with clean, well characterised, single crystal surfaces of aluminium, silicon and chromium. The characterisation by LEED and Auger techniques is most important in surface experiments as contaminants can easily cover a surface and obliterate all of the clean surface properties. The energies of positrons re-emitted from the surface were analysed by applying a retarding field to the sample and measuring the increase in the number of positrons annihilating inside the sample. The spectra had two components: a sharp peak at very low energies due to positrons that had thermalised inside the solid, and a much broader distribution due to incompletely thermalised positrons. The latter component became more intense as the incident energy was lowered below $1000 \mathrm{eV}$, a consequence of decreasing penetrating power.

The sharp peak was seen in all the materials studied, beginning its rise at zero kinetic energy and having a width dependent on the solid: $0.5 \mathrm{eV}$ for aluminium, $1.0 \mathrm{eV}$ for silicon and $1.7 \mathrm{eV}$ for chromium. Because the positrons in this peak have thermalised inside the solid, the peak cuts off at a maximum energy which is the "negative affinity' the solid has for positrons.

Our understanding of positrons in solids and at surfaces is based on calculation and on some experimental annihilation rates and angular distributions. The new experiments will provide a more detailed picture of positron behaviour. Theories of the positronsolid force law such as that of Hodges \& Stott (Solid State Commun. 12, 1153 ; 1973) can now be checked, even to the extent of diffracting positrons from surfaces. The rate of energy loss of positrons in solids can be determined from the ratio of thermalised to unthermalised positrons emitted: accurate calculations are difficult especially when the positron is moving slowly. Some new problems present themselves. The width of the low energy emission peak is only of the order of $1 \mathrm{eV}$ but this is still more than an order of magnitude larger than the thermal spread in energies. Simple theoretical arguments require that the positrons are accelerated normal to a perfect surface, and if inelastic collisions are excluded, emerge in a parallel beam with only a thermal spread of energies. Certainly this is the situation found for electrons in negative electron affinity systems. The emission process for positrons must be more complicated and is yet to be elucidated.

Finally, we can look forward to the more difficult experiment of detecting emission of positronium. Canter, Berko and Mills (Phys. Rev. Lett. 33, 7; 1974) have shown that a large fraction of the positrons pair with electrons as they leave the solid and emerge as positronium. This pair-tunnelling process reminiscent of the interface between a normal and superconducting metal can be studied by measuring the angle and energy distribution of the ejected positronium.

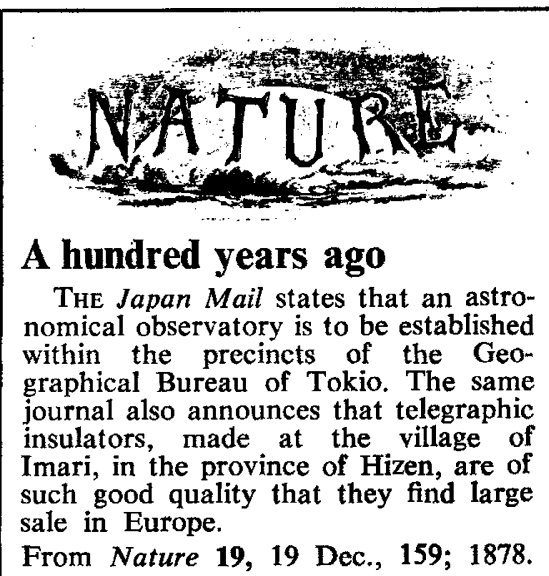

